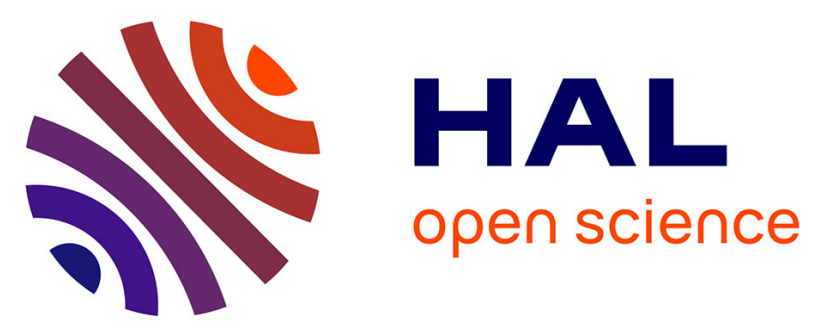

\title{
End-User Visual Design of Web-Based Interactive Applications Making Use of Geographical Information: the WINDMash Approach
}

The Nhan Luong, Patrick Etcheverry, Thierry Nodenot, Christophe Marquesuzaà, Philippe Lopistéguy

\section{To cite this version:}

The Nhan Luong, Patrick Etcheverry, Thierry Nodenot, Christophe Marquesuzaà, Philippe Lopistéguy. End-User Visual Design of Web-Based Interactive Applications Making Use of Geographical Information: the WINDMash Approach. The 5th European Conference on Technology Enhanced Learning, Sep 2010, Barcelona, Spain. pp.536-541, 10.1007/978-3-642-16020-2_50 . hal-00634779

\author{
HAL Id: hal-00634779 \\ https://hal.science/hal-00634779
}

Submitted on 23 Oct 2011

HAL is a multi-disciplinary open access archive for the deposit and dissemination of scientific research documents, whether they are published or not. The documents may come from teaching and research institutions in France or abroad, or from public or private research centers.
L'archive ouverte pluridisciplinaire $\mathbf{H A L}$, est destinée au dépôt et à la diffusion de documents scientifiques de niveau recherche, publiés ou non, émanant des établissements d'enseignement et de recherche français ou étrangers, des laboratoires publics ou privés. 


\title{
End-User Visual Design of Web-Based Interactive Applications Making Use of Geographical Information: the WINDMash Approach
}

\author{
The Nhan Luong1, Patrick Etcheverry1, Thierry Nodenot1, \\ Christophe Marquesuzaà1 and Philippe Lopistéguy1 \\ 1IUT de Bayonne - Pays Basque, LIUPPA-T2I, 2 Allée du Parc Montaury \\ 64600 Anglet, FRANCE \\ \{thenhan.luong, patrick.etcheverry, thierry.nodenot, christophe.marquesuzaa, \\ philippe.lopisteguy\}@ iutbayonne.univ-pau.fr
}

\begin{abstract}
Visual instructional design languages currently provide notations for representing the intermediate and final results of a knowledge engineering process. This paper reports on a visual framework (called WIND - Web INteraction Design) that focuses on both designers' creativity and model executability. It only addresses Active Reading Learning Scenarios making use of localized documents (travel stories, travel guides). Our research challenge is to enable the teachers to design by themselves interaction scenarios for such a domain, avoiding any programmer intervention. The WIND framework provides a conceptual model and its associated Application Programming Interface (API). The WIND interaction scenarios are encoded as XML documents which are automatically transformed into code thanks to the provided API, thus providing designers with a real application that they can immediately assess and modify (prototyping techniques). The WIND conceptual model only provides designers with an abstract syntax and a semantics. Users of such a Domain Specific Language (DSL) need a concrete syntax. Our choice is to produce a Web-Based Mashup Environment providing designers with visual functionality.
\end{abstract}

Keywords: Interaction Design, Semantic Web, Applications of TEL in the Domain of Geography, Visual Instructional Design Languages, Technologies for Personalisation and Adaptation.

\section{Introduction}

Research works dedicated to Visual Instructional Design Languages (VIDL) are evolving rapidly. This article reports on our current research cycle which aims at promoting a visual language (called WIND) that focuses on both designers' creativity and model executability. The WIND language only addresses Active Reading Learning Scenarios. This specific pedagogical activity "refers to set of high level reading, searching, problem solving and meta-cognitive skills used as readers proactively construct new knowledge" [1], making use of localized documents (travel 
stories, travel guides) that embed a lot of geographical information about the movements of an actor within a territory. Our research challenge is to provide teachers (user-designer) with convenient instruments in order to design and to assess by themselves interaction scenarios for such a domain, avoiding any programmer intervention.

Our research team has developed for three years:

- a set of tools and software components for the automatic tagging of Geographical Information within textual documents. Such geographical information is composed of three complementary features: the spatial feature (SF), the temporal feature (TF) and the phenomenon feature [2]. The "spatial chain" produces an index where each SF is associated with one or more geometries. Similarly, the "temporal chain" associates TF to one or more temporal intervals and the "thematic chain" is based on well-known statistical criteria (terms frequency). Currently, two versions of such automatic chains are exploited: GeoStream [3] is a web service that can tag $\mathrm{SF}$ within textual documents, while $\pi \mathrm{R}$ [4] is another web service that can tag movement verbs and SF in order to find itineraries within specific textual documents (travel stories, travel guides).

- a set of tools and software components (text component, map component, calendar component) that can be parameterized and combined [5] with previous web services in order to author dedicated applications favouring active learning scenarios in relation with the discovery of a territory (see http://erozate.iutbayonne.univ-pau.fr/forbes2007/exp/ for an example). We set up two specific features of these educational applications compared with currently available web-based cartographic applications:

1. The focus is on interaction and not on data visualization;

2. The map is no longer the central component, neither is the text, nor the calendar: the user (learner) needs to interact from any of these components and the system should react on any of these components.

The experience gained about the production of such applications led us to initiate two complementary research actions focusing on empirical visual design approaches. The first one consists in developing a Mashup environment [6] enabling a pedagogue (teacher) to handle by him/herself the elicited modules to design and to assess his/her pedagogical application. He/She is thus able to retrieve/select travel stories or travel guides extracts, to imagine dedicated learning scenarios (how the learner may control the interaction with the application, which messages are provided to the learner) and to build the user interface (how it is organized and which interactions are available between the different parts of the interface). The second research action focuses on the design tasks required to enable a pedagogue describing how the system will diagnose the learner's behaviour while using such interactive application in order to solve a particular quiz or problem.

In this paper we shall focus on the first action but both actions should enable a teacher to easily formalize and to evaluate his/her educational ideas by using (as a learner) the automatically generated application. We therefore promote an agile design approach (evaluation step should therefore be used to check/criticize/confirm previous pedagogical choices) made possible thanks to "agile" design tools that should fully imply the end-user along the whole process by rapidly integrating his/her 
requirements into a technical solution.

In the next section, we present WINDMash, a web mashup environment that designers can use both to create and to assess interactive scenarios that handle geographical information. Last, discussion recalls WIND capabilities and our current works to improve the WIND framework usability.

\section{WINDMash, an Environment Dedicated to the Authoring of Active Learning Scenarios for Territory Discovery}

This section describes WINDMash, a mashup environment that designers can use both to create and to assess interactive scenarios that handle geographical information. WINDMash provides designers with an authoring and an execution framework that promote an agile approach to shorten as much as possible the delay between the design and the evaluation step of an interactive application. Such an approach is required now to favour end-user modelling. Let us consider a WIND application whose learning objective is to help the learners to discover prefecture around the cities that the user may highlight in the text area ${ }^{1}$ :

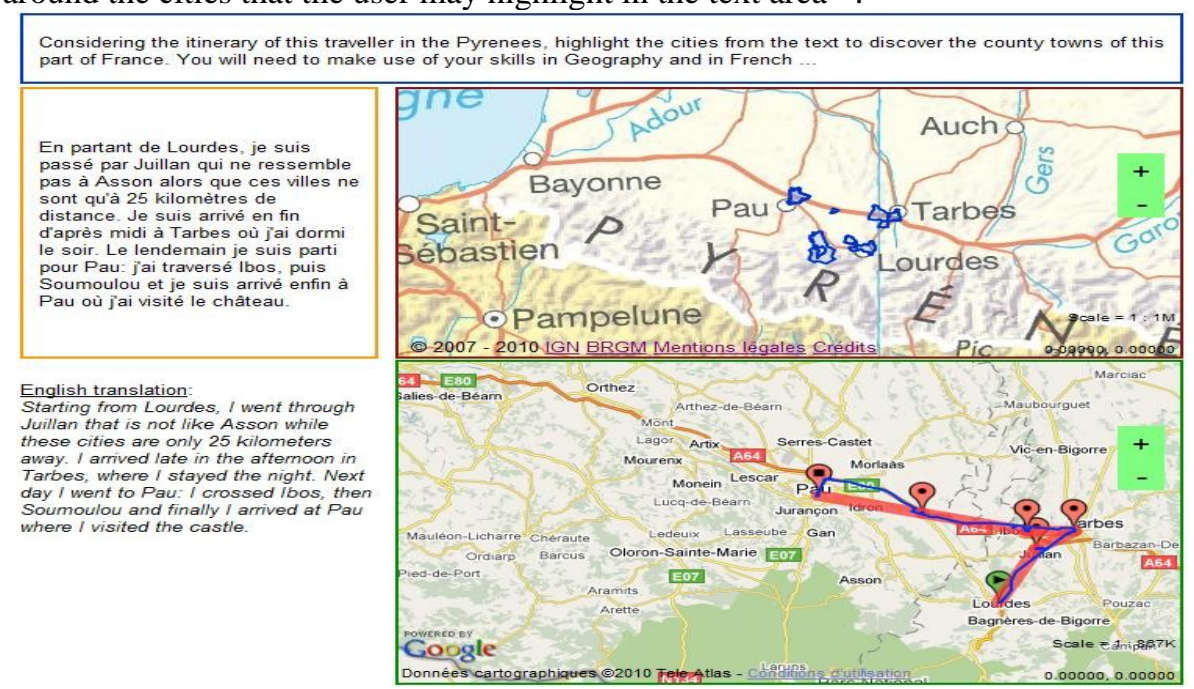

Fig. 1. An example (see http://erozate.iutbayonne.univ-pau.fr/Nhan/ectel2010/example.html) for educational purpose.

We advocate that, using WINDMash, a designer (teacher) without any computer science skills can easily describe and assess such an interactive application. The instructional design process promotes three design facets (data, interface and interaction) in order to generate an educational web application from initially informal requirements.

1 Within the text, there are many words referring places, some of them are cities, others are not (e.g. the Adour river or the Ossau Valley). If the user highlights a city (e.g. the city of Lourdes), the map next zooms on the prefecture around such a city (e.g. the prefecture of Lourdes is Tarbes); in the other cases (rivers, valleys, mountains, etc.), a message is sent to the user. 


\subsection{The Data Facet}

The data facet focuses on the information that will be provided to the learner at runtime. Starting from one or from several plain texts, the designer (teacher) may easily create a processing chain by selecting dedicated modules. This processing chain can automatically transform such input into results that can be either processed again or can be visualised with dedicated viewers: text, map, and calendar viewers (cf. Fig. 2). Available modules can be parameterized by the designer to reach a specific goal, enabling the designer:

- to normalize plain texts into the WIND format;

- to extract places, itineraries, etc;

- to intersect or to join previous results;

- to later visualize results with dedicated viewers to check the design process.

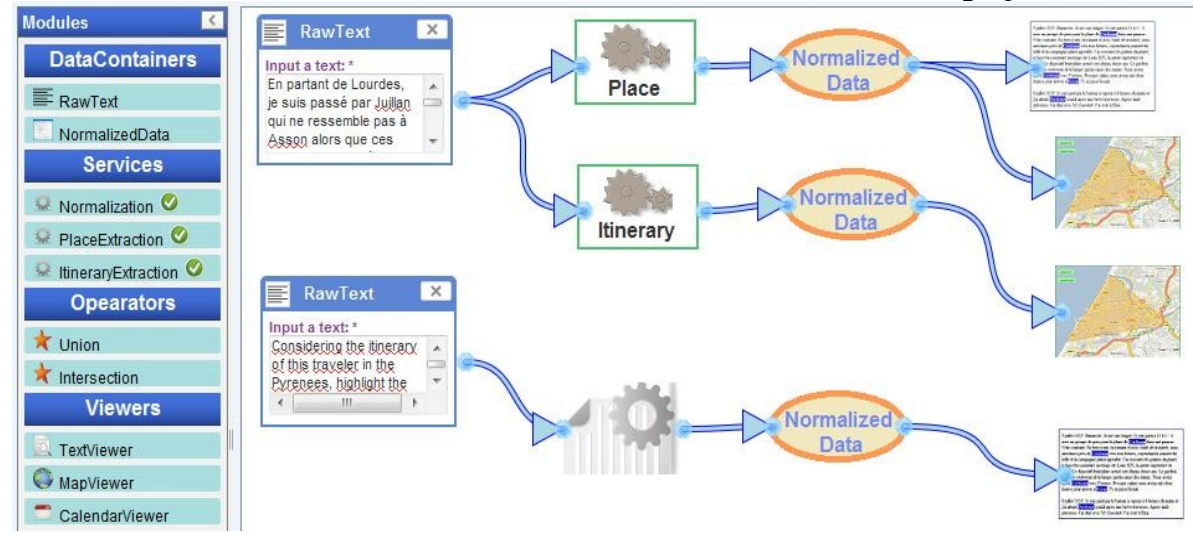

Fig. 2. Screenshot example for the data facet of the WINDMash environment.

These modules are described in two ways: (1) as JSON format (see http://erozate.iutbayonne.univ-pau.fr/Nhan/windmash/modules.js) to facilitate the implementation of the environment; (2) as description texts (comprehensible by the designers) when the designers hold mouse over the modules.

\subsection{The Interface Facet}

The interface facet enables the designers to organize the interface of the generated application (size, position, map provider, zoom level...). The Viewers from the previous facet are concerned here. An interface containing all the Viewers is automatically generated and displayed to the designer, enabling him/her easily and rapidly define the look and feel of each Viewer. Each Viewer displays the information from the data facet and the designer may then decide where each Viewer should be presented on the screen by clicking on its header and by dragging onto a new position; he/she can also easily resize it. Each Viewer has also specified characteristics. For example: 
- For a TextViewer, the geographical words (TextPart) are automatically tagged by the Service modules of the data facet.

- For a MapViewer, the MapParts are automatically marked as geometries on the map layer. A point represents a location, a place; a line represents a route, a river, an itinerary; a polygon represents a region, a city, etc.

- For a CalendarViewer, the concerned time (CalendarPart) may be tagged and displayed.

\subsection{The Interaction Facet}

This facet allows the designer (teacher) to design the interactions between the viewers displayed in the previous facet. Currently, by default, we automatically offer some interactions between the TextViewer, the MapViewer, and the CalendarViewer:

- when clicking on the TextPart, the corresponding MapPart is focused and the corresponding CalendarPart is highlighted;

- when clicking on the MapPart, the corresponding TextPart is boldfaced and the corresponding CalendarPart is highlighted;

- when clicking on the CalendarPart, the corresponding TextPart is boldfaced and the corresponding MapPart is focused.

\section{Discussion and Future Directions}

In this paper, we presented WINDMash, our Mashup environment that demonstrates current capabilities of the WIND framework and its API. This environment focuses on the design of geographical applications making use of specific localized documents called "travel stories". As soon as a step is completed, the designer can execute the code which is automatically generated thanks to the WIND API. Thus, at each step of the design process (data, interface and interaction facets), it is possible to immediately visualize the design results without having to know anything about the underlying Web Mapping Services (IGN Geoportail API, Google Maps API, OpenLayers API, etc.). Of course, if, at runtime, something appears to fail, it is very easy to modify what was specified at any design step.

WINDMash can automatically extract the geographical information (place extraction, itinerary extraction) contained in such textual documents: this is the WINDMash data facet. From such information (or any inferred information from this latter information and our geographical ontology), designers can describe the interface of the application composed of Map / Text / Calendar visual components: this is the WINDMash interface facet. Our ongoing works consist in the WINDMash interaction facet. We need to focus on a visual language to describe the interactions between the visual components.

The WINDMash (see http://erozate.iutbayonne.univ-pau.fr/Nhan/windmash/) environment is currently still work in progress, according to our advances about: 
- Semantic web techniques particularly for the automatic annotation of geographical information embedded in texts.

- Formal and semi-formal techniques for Human Computer Interaction: extension of the UML sequence diagram formalism to exploit both the geographical semantics captured within texts and text/map/calendar viewers available functionality; delayed reactions of the application according to decisional state-diagrams descriptions.

- Experiments of the WINDMash toolset by both pedagogues and learners. From the beginning of these works, we cooperate with teachers of several classrooms and colleges to evaluate with their pupils [7] the educational potentiality of the microworlds that we can design with the WIND framework. Teachers also help us to go further in order to be able to embed these microworlds into educational activities described in terms of learning goals and cognitive tasks needed to solve a problem, tutor goals, ...

\section{Acknowledgements}

This research is supported by the French Aquitaine Region (project $n^{\circ} 20071104037$ ) and the Pyrénées-Atlantiques Department ("Pyrénées : Itinéraires Educatifs" project).

\section{References}

1. Murray, T., Hyperbook Features Supporting Active Reading Skills. Chapter 8 in Web-based Intelligent e-Learning Systems: Technologies and Applications, by Zomgmin Ma (Ed.). Idea Group Publishing: Hershey, PA, pp. 156-174. (2005)

2. Gaio, M., et al., A Global Process to Access Documents' Contents from a Geographical Point of View. Journal of Visual Languages and Computing (JVLC), 2008. 19(1): p. 3-23.

3. Sallaberry, C., et al., GeoStream: a Spatial Information Indexing Web Service, in First International Opensource Geospatial Research Symposium (OGRS 2009), Springer, Editor. 2009: Nantes (France).

4. Loustau, P., T. Nodenot, and M. Gaio, Design principles and first educational experiments of PIIR, a platform to infer geo-referenced itineraries from travel stories. International Journal of Interactive Technology and Smart Education (ITSE), 2009. 6(1): p. 23-39.

5. Luong, T. N., et al., WIND: an Interaction Lightweight Programming Model for Geographical Web Applications, in First International Opensource Geospatial Research Symposium (OGRS 2009), Springer, Editor. 2009: Nantes (France).

6. Taivalsaari, A., MashWare: The Futur of Web Applications. Technical Report (2009).

7. Luong, T. N., et al., A framework to author educational interactions for geographical web applications. In Fourth European Conference on Technology Enhanced Learning (EC-TEL 2009), pages $769-775$. 